\title{
Perspectivas teóricas de género: status questionis del impacto en el sistema educativo
}

\author{
Theoretical perspectives on gender: status quaestionis of the impact \\ on the educational system
}

\author{
Ana Sánchez-Bello/ana.sanchez.bello@udc.es \\ Universidade da Coruña, España
}

\begin{abstract}
Gender studies in social sciences are influenced, in various manners and with diverse consequences as well, by the different perspectives of feminist theory. The traditional classification alludes to four tendencies of the feminist theory; nowadays however, the tendency of the postmodern, the difference and queer theories have been incorporated. These are the agglutinating theoretical proposals among the multiplicity of tendencies we can find at present. This work carries out a theoretical revision on these proposals in order to analyze which aspects develop each one of them in relation to their contribution to the educational system. It is concluded that these proposals have to be analyzed as allies, not as opposed, for the objective of all of them is to find various solutions to different problems in the educational sphere
\end{abstract}

Key words: gender theory, educational system, women's studies, equity, science.

Resumen: Los estudios de género en las ciencias sociales están influidos, de diverso modo y con consecuencias también diversas, por las diferentes perspectivas que se presentan en la teoría feminista. La clasificación clásica hace alusión a cuatro tendencias de la teoría feminista, pero en la actualidad se ha incorporado la tendencia posmoderna, de la diferencia y Queer. Éstas son las propuestas teóricas aglutinadoras de entre la multiplicidad de tendencias que podemos encontrar en la actualidad. Este trabajo realiza una revisión teórica sobre dichaspropuestas para, en segundo lugar, analizar qué aspectos desarrollan cada una de ellas en relación con su aportación al sistema educativo. Se concluye que estas propuestas deben ser analizadas como aliadas y no como opuestas, ya que el objetivo de todas ellas es encontrar soluciones diversas a problemas diversos en el ámbito educativo.

Palabras clave: Teoría de género, sistema educativo, estudios de las mujeres, equidad, ciencia. 


\section{Introducción}

Los estudios de género, desde los años setenta, han supuesto la incorporación de una de las categorías analíticas de mayor influencia en las ciencias sociales, insertándose en el interés emancipatorio descrito por Jürgen Habermas (1982). Es especialmente destacado el trabajo realizado en el marco de la epistemología, ${ }^{1}$ al poner en tela de juicio la categoría de sujeto cognoscente y, al mismo tiempo, cuestionar fuertemente la categoría clásica de objetividad (Anderson, 2012).

El género, como categoría analítica en las ciencias sociales, comenzó a implantarse en los años sesenta en las universidades americanas a través de los Women's Studies, cuyo interés radicaba en que el conocimiento científico debía poseer una estrecha vinculación con la vida de las mujeres, hacer ciencia tenía que ser un acto de mejora de la vida de los individuos. De este modo, la incorporación de estos estudios al conocimiento científico social ha tenido como consecuencia la reformulación de los principios de la tradición científica desde un doble cometido; por un lado, analizar críticamente las construcciones teóricas patriarcales, y por otro, acunar nuevos conceptos que ayuden a explicar la realidad en su totalidad, introduciendo aspectos que, hasta el momento, no habían sido tenidos en cuenta, como por ejemplo: el espacio privado, la violencia contra las mujeres, la división sexual del trabajo, los estereotipos sociales sobre el género o la feminización de la pobreza, entre otros. Los estudios de género han puesto de relieve cómo la ciencia posee sesgos que implican perspectivas parciales basadas en el androcentrismo. La constitución de la ciencia bajo el paraguas androcéntrico ha supuesto la estructuración del pensamiento en categorías dicotómicas como son: público vs privado; objetividad vs subjetividad; razón vs sentimiento. Dichas categorías se asientan sobre la parcelación de la realidad bajo otra división que las engloba:

1 Dos autoras de referente obligado para poner en cuestión la objetividad científica desde la Teoría de género son Jane Flax (1981) y Sandra Harding (2002, 2010a). La primera cuestiona la objetividad "clásica" porque se basa en la diferenciación entre sujeto de conocimiento y objeto. La validez científica parecía centrarse en la autonomía del sujeto cognoscente con el objeto por investigar. Sandra Harding también realiza una pormenorizada crítica a la objetividad "tradicional", afirmando que la inclusión en la ciencia de las interpretaciones de los grupos que no han estado históricamente representados en la investigación científica asegura la consecución de un mayor nivel de objetividad. Otra de las autoras más referenciadas es Helen Longino (2002), cuyo trabajo, aunque no pone el acento en la cuestión de la objetividad, ha sido ampliamente difundido por sus aportaciones a la relación entre razonamiento y los estándares de la comunidad, lo que influye en la consideración sobre la objetividad. 
masculino $v$ f femenino, lo que ha llevado a establecer juicios de valor sobre el papel social de cada uno de ellos, siendo las concepciones adscritas al género lo que sitúa "el punto de vista" como el cimiento para comenzar a analizar la dicotomía social, mediante las diferencias culturales y de relaciones de poder (Harding, 2010b).

Los estudios de género están muy vinculados al contexto de lucha por la democracia, ya que ésta significaba, entre otras muchas cosas, el reconocimiento de la participación en la vida pública de grupos sociales que hasta el momento habían estado invisibilizados en el debate social. Entre ellos destaca el movimiento de mujeres que comienza a ser reconocido, en un primer momento, por su poder de presencia pública y posibilidad de voto (Hobson, 2003: 64). Estos grupos son muy conscientes de la importancia de la incorporación del género en el sistema escolar para que los ciudadanos y ciudadanas del futuro posean las herramientas necesarias para ejercer el derecho democrático en toda su plenitud.

Desde esta interpretación inclusiva, la escolarización debe cumplir el papel fundamental de trabajar por la consecución de la igualdad de oportunidades, diluyendo las discriminaciones por razón de sexo que impiden el desarrollo personal y comunitario. Este derecho es sólo posible si se adquieren estrategias individuales para desenvolver la autonomía de pensamiento y, por lo tanto, de acción. Entre las demandas más destacadas está la de modificar los contenidos y la práctica en el sistema educativo con el objetivo de introducir mecanismos de igualdad real en el proceso educativo.

Estas demandas están influidas, de una u otra manera, por las diferentes perspectivas que se presentan en la teoría feminista. Alison Jaggar en su obra Feminist, Politics and Human Nature, publicada en 1983, clasificaba en cuatro las tendencias de la teoría feminista: liberal, socialista, marxista y radical, aunque posteriormente se generalizará la unión entre socialismo y marxismo, quedando de esta manera la clasificación en tres tendencias. Las últimas incorporaciones más destacadas son las tendencias posmodernas, de la diferencia y Queer; sintetizando así las propuestas teóricas que aglutinan a la multiplicidad de tendencias vigentes que podemos encontrar en la actualidad (Lorber, 2010: 21).

En este texto se describirán cuáles son las bases argumentales en las que se postulan las diferentes perspectivas teóricas y cuál ha sido la aportación que han realizado a la educación formal, es decir, se examinará el impacto de género de las mismas en el sistema educativo. El análisis sobre este impacto comienza con el estudio sobre las fundamentaciones teóricas que se han 
llevado a cabo en torno al género y que aglutinamos alrededor de las cuatro perspectivas ya señaladas. Cada una de ellas posee una conceptualización sobre la consideración ontológica del ser mujer y, por lo tanto, su papel en la sociedad y sus dificultades para la incorporación al espacio público en igualdad de condiciones que los varones. En estrecha vinculación se examinan también las propuestas que, desde el ámbito de la educación formal, ofrecen cada una de las distintas perspectivas para dar solución a las situaciones de desventaja que sufren las mujeres.

Podremos descubrir que, ante la diversidad de principios teóricos, se han llevado a cabo propuestas y experiencias muy variadas con un mismo objetivo: conseguir la mejora social para las mujeres. Desde este principio, en el sistema escolar se ha desarrollado una gran variedad de alternativas prácticas en torno a la equidad desde una interpretación holística, debido a que existe una multitud de factores interrelacionados que afectan a la desventaja social de las mujeres.

Los apartados siguientes analizan cada una de las perspectivas en torno a la consideración específica de las causas de subordinación de las mujeres y las propuestas educativas que cada una de ellas presenta para enmendarlas.

\section{Las barreras formales en educación: la perspectiva liberal}

La perspectiva teórica liberal está basada en una concepción individualista del ser humano, es decir, lo considera como agente y responsable último de lo que suceda; por lo tanto, el papel del Estado se pone en cuestión; se anteponen los intereses individuales a los colectivos. El feminismo de raíz liberal afirma que a las mujeres se les impide ser agentes de su propia individualidad y, por lo tanto, no poseen la libertad individual que poseen los varones, debido a que no tienen las mismas oportunidades de partida en casi ninguno de los órdenes sociales, lo cual las sitúa en desventaja en comparación con el sexo masculino. La razón que observan en las causas de la subordinación femenina es la falta de garantías de igualdad tanto a nivel legislativo como político, laboral, emocional o familiar, por ello postulan que para garantizar la igualdad sexual es necesario, en primer lugar, abolir las leyes y políticas discriminatorias por razón de sexo y, en segundo lugar, modificar la diferenciación de roles que existe en la sociedad y que se observa en la división sexual del trabajo y en la dicotomización sexual de los espacios.

La obra de Betty Friedan visibilizará todo este conglomerado de situaciones de desigualdad que viven las mujeres (Wood, 2011) y que se concreta en la famosa frase: "El problema que no tiene nombre", haciendo alusión al 
descontento de las mujeres de clase media americana que eran incapaces de interpretar su propia situación de desigualdad.

Las representantes de esta tendencia consideran que el sistema educativo es el que posee mayor capacidad para solucionar los problemas de desventaja social que sufren las mujeres; para ello, se debería garantizar que las chicas tengan las mismas oportunidades que los chicos, que no exista ningún obstáculo para el acceso a cualquier titulación o para la elección de asignaturas en cualquier nivel educativo.

Las dificultades de género no se refieren únicamente a cuestiones legislativas sino también a situaciones relacionadas con el aprendizaje de estereotipos. Combatir los estereotipos sexistas ha sido el campo de batalla (junto con la igualdad de acceso a todos los niveles y opciones académicas) en el que han puesto más ahínco las representantes liberales. El mayor éxito de la perspectiva liberal se ha dado en lo relativo a las barreras formales que tienen las mujeres para el acceso al sistema educativo, pero ha sido mucho menor en los procesos informales, como, por ejemplo, las implicaciones emocionales de las decisiones de mujeres que las mantienen en situaciones de subordinación y cómo el sistema educativo no actúa ante situaciones de violencia explícita o implícita, de los procesos de coacción no reglamentarios.

La escuela es un buen ejemplo del éxito de la perspectiva liberal, el cual se puede apreciar en los datos de ingreso de las mujeres en las titulaciones universitarias, ${ }^{2}$ sin obviar que sigue existiendo una "sexualización" de las carreras universitarias y de los estudios de formación profesional ${ }^{3}$, así como en la "feminización vertical" ${ }^{4}$ de la función docente. En estos aspectos la pers-

2 Según las cifras del Ministerio de Educación de España, en este país, en el curso 20092010 las mujeres representan un 54,2\% del total del alumnado universitario y un $47,34 \%$ en formación profesional.

3 Según las cifras del mismo organismo y para el mismo curso académico, las alumnas matriculadas en Artes y Humanidades representan un 61,4\% del total del alumnado, y en Ciencias de la Salud un 72,1\%; mientras que en las titulaciones de Ingeniería y Arquitectura el porcentaje desciende hasta un $26,8 \%$. En la formación profesional, la desigualdad sexual en la elección de estudios es muy relevante. Se observa, según la misma fuente, que un 95,42\% del alumnado matriculado en la especialidad de Imagen Personal son mujeres, y en Servicios Socioculturales a la Comunidad un $86,83 \%$. Las cifras se invierten en titulaciones como Electricidad y Electrónica con un 2,65\% de alumnas o Mantenimiento de Vehículos con un $2,80 \%$ de estudiantes femeninas.

4 La desigualdad vertical se manifiesta cuando el número de mujeres con cargos de responsabilidad (mejor dotados económicamente y más valorados socialmente) es menor que la 
pectiva liberal no ha conseguido todavía sus objetivos, por ello sigue haciendo hincapié en que la socialización en los roles sexuales sigue siendo un remedio muy efectivo para lograr sus propósitos.

Las críticas a la tendencia liberal hablan de la ausencia de cuestionamiento del modelo social imperante, el cual posee valores construidos desde la masculinidad como la competitividad o el individualismo. Esto conlleva a que las niñas han de aprender el modelo masculino si quieren tener éxito, en lugar de cuestionarse qué modelo defiende la escuela y si debe ser modificado, potenciando otro tipo de valores adjudicados históricamente a las mujeres.

Además, como hemos observado, la igualdad en el acceso a la educación no se ha conseguido todavía. Las diferencias entre las diversas alternativas que oferta la formación profesional sigue estando muy influida por los estereotipos de género en función de los oficios, la misma dicotomía se encuentra en las carreras universitarias, pues siguen existiendo estudios superiores feminizados y otros, por el contrario, donde acuden mayoritariamente los chicos.

\section{La docencia en femenino al servicio del capital: la perspectiva socialista}

La perspectiva socialista-marxista hace una crítica abierta al modelo patriarcal de desigualdad entre varones y mujeres desde una interpretación economicista, aunque, al mismo tiempo, estas autoras cuestionan que la opresión de clase sea la única opresión sufrida por las mujeres. Dicha perspectiva se desarrolló fundamentalmente a finales de los años setenta y principios de los ochenta de la mano de autoras tan representativas como Zillah Eisenstein, Heidi Hartmann o Juliet Mitchell (Sánchez et al., 2001), quienes afirmaban que la erradicación de la opresión de las mujeres supondría una modificación de la estructura social con consecuencias económicas, políticas, culturales, ideológicas, etc. En este sentido, la autora que hablará abiertamente de patriarcado capitalista será Zilla Eisenstein (1980), al desarrollar lo que se ha denominado Teoría del doble sistema, según la cual la desigualdad de las mu-

tasa masculina. Este eje transversal es el que ha obligado a redefinir el término "trabajo" en los diferentes análisis e investigaciones que se están realizando en las dos últimas décadas desde una perspectiva de género y que ha influido en la sociología del trabajo, reconociendo que el trabajo es mucho más "que la relación social de empleo" (Giner, Lamo de Espinosa y Torres, 1998). Es ya un hecho, en la comunidad científica, la incorporación de las actividades productivas no remuneradas en la conceptualización sobre el trabajo. 
jeres no estaba causada exclusivamente por el sistema capitalista, al constatar que dicha opresión era interclasista. Esto sugería la existencia de factores más allá de los económicos que influían en la desigualdad de género.

Esta tendencia de corte socialista en el ámbito educativo ha realizado destacadas modificaciones en sus tesis sobre educación y feminismo en los últimos cuarenta años. En la década de 1960 este movimiento se interesaba por demostrar cómo la situación de las mujeres en el ámbito educativo estaba al servicio del sistema económico al perpetuar la división sexual del trabajo (tanto vertical como horizontal) de las profesoras, tal y como señaló Maggie Coats (1994). Al mismo tiempo, las alumnas aprendían en la escuela a reproducir el sistema patriarcal vigente que consistía, básicamente, en el aprendizaje de los roles femeninos, a través de una enseñanza androcéntrica.

Posteriormente, a partir de los años ochenta, el feminismo socialista reconoce que las necesidades del capital son mucho mayores que las exclusivamente económicas y se vuelca en señalar que la escuela ha de trabajar para que el alumnado comprenda cómo funciona la estructura social para favorecer la opresión de las mujeres. En este sentido, esta perspectiva modifica las tesis clásicas del marxismo al introducir la opresión de las mujeres como una opresión específica, no haciendo depender el patriarcado de la estructura económica, sino de una relación entre ambas que se apoyan y se necesitan mutuamente.

La relación entre capitalismo y desigualdad de género se explica porque este modelo económico y político utiliza a las mujeres como mano de obra barata en dos frentes: por un lado, en el espacio público, abaratando los costes y, por otro, en el espacio privado, al realizar el trabajo doméstico y familiar gratuitamente (Holmstrom, 2002). La reconceptualización del trabajo doméstico y la reproducción han sido los ejes principales sobre los que ha girado el pensamiento feminista socialista durante los setenta y ochenta. A partir de los noventa, se ampliará su campo de intervención hacia el análisis de la prostitución y la pornografía como ámbitos de opresión patriarcal sobre las mujeres.

Según esta perspectiva teórica, la institución escolar está al servicio del sistema capitalista y éste es patriarcal. Los centros de enseñanza, por lo tanto, contribuirían a fomentar la desigualdad sexual, porque las chicas eligen carreras típicamente femeninas que, además, son más baratas para el Estado y, al mismo tiempo, sirven para hacer que éstas trabajen también en el hogar. Por ello, el sistema educativo fomentaría la asociación de ciertas carreras con el rol de madre, de cuidadora de la familia. Para dar solución a dicho problema, esta perspectiva afirma que se debería modificar el aprendizaje sexuado de los 
roles, pero, al mismo tiempo, debería introducir una perspectiva crítica en el currículum y, en especial, en los contenidos culturales que vehiculizan las distintas asignaturas; un currículum que obligue a tomar en consideración el papel de la estructura social y cuál es el que las mujeres juegan en ella.

En este sentido, se apuesta por modificar el papel que desempeña alumnado y profesorado. Las estudiantes toman parte activa en el proceso de enseñanza-aprendizaje, no siendo meras receptoras de información; sino por el contrario, contribuyen al aprendizaje del resto de las personas del aula con las aportaciones sobre sus experiencias. Es decir, convierten a las alumnas en generadoras de conocimiento, pudiendo modificar las ideas previas sobre lo que son, sienten y piensan las propias mujeres.

Es una perspectiva teórica eminentemente política, lo cual le lleva a hacer contacto con autores como Michael Apple, Henry Giroux, Peter McLaren, Paulo Freire o Ira Shor (Totten y Pedersen, 2012). Estos autores han puesto especial relevancia en cuestiones de opresión de diferentes grupos sociales, aunque no hacen referencia específica a la desigualdad de género, como sí lo harán las teorías denominadas feministas socialistas. A los autores y autoras del pensamiento pedagógico crítico y feminista socialista les une su indudable base neomarxista, que han modificado y reinterpretado para comprender mejor el sistema educativo y sus interacciones con otras esferas de la sociedad.

\section{El patriarcado como principio analítico en educación: la perspectiva radical}

La perspectiva radical surge en paralelo al encumbramiento del movimiento de liberación de las mujeres de los años sesenta y de la nueva izquierda americana de inspiración marxista como, por ejemplo, las representantes: Kate Millet, Catharine MacKinnon o Sulamit Firestone, cuyos textos han pasado a la historia como obras fundacionales (Kolmar y Bartkowski, 2010) que centran la causa de la opresión de las mujeres en la instauración de una sociedad basada en el patriarcado (término que ellas mismas acuñaron). Entienden que el patriarcado es un conflicto histórico que ha jugado a favor de los varones. Para esta perspectiva, el problema de la opresión femenina se centra en cuestiones como: la violencia contra las mujeres (basada en la dependencia emocional y afectiva de las mujeres), la valoración estereotipada de la identidad femenina y cuestiones de interpretación de la sexualidad basadas en la utilización del cuerpo femenino como objeto. 
Las propuestas del "radicalismo" en el ámbito de la enseñanza están vinculadas con dos acciones globales: el trabajo con el ámbito de los sentimientos y la autovaloración positiva de las mujeres. En relación con la primera de ellas, se trataría de utilizar el cuidado hacia los otros no sólo como una parte del aprendizaje de las mujeres, sino extrapolarlo a toda la humanidad, pues esta tendencia considera que si también los varones desarrollasen aprendizajes de cuidado y atención, se conseguirían menores niveles de violencia entre los varones, desarrollando así una masculinidad más ligada a la vida y menos a los actos violentos (Connell, 2001, 2008).

Desde esta misma consideración, se apela a la necesidad de que los chicos también trabajen con sus sentimientos, siendo capaces de expresar tristeza, frustración, descontento, etc., con el objetivo de que explorar estas emociones en el sistema educativo sirva para rebajar el nivel de violencia masculina y construir una sociedad más equitativa (Renau, 2012).

Desde la idea de la importancia de la autovaloración de las mujeres, su trabajo en la escuela se centra en modificar la opresión debida a la cultura sexista, haciendo especial hincapié en los procesos de concienciación (Weiner, 1999: 153). Esto implica dar voz a las mujeres para que puedan reconstruir su propia historia personal, reconstruir positivamente la identidad femenina y fortalecerlas con el fin de una búsqueda de un cambio social con nuevos valores sociales. Al mismo tiempo se trabajaría en valorar el punto de vista femenino de cualquier ámbito del currículum escolar, como por ejemplo: historia, filosofía, literatura, ciencias, etc. Así como la visibilización de las mujeres en los contenidos que son trabajados en los centros escolares.

Paralelamente, uno de los aspectos en los que más atención se puso, en la investigación y la práctica desarrolladas en los centros escolares, fue en la erradicación de los roles de género, siendo el campo de actuación más prolífero de la pedagogía feminista en los años setenta y ochenta (Skelton y Francis, 2009: 11). Desde los movimientos de mujeres se consideraba que el patriarcado utilizaba los estereotipos como estrategia de invisibilización de las capacidades de las mujeres, y, por lo tanto, se entendía que con la modificación de estos moldes se conseguiría el proyecto de liberación de parte de ellas respecto al orden patriarcal. La generalización de un modelo ontológico sobre las mujeres es considerado el primer mecanismo ideológico que reproduce y, al mismo tiempo, refuerza las desigualdades de género (Cobo, 2001: 15). El cuestionamiento de las capacidades de las mujeres para integrarse en el ámbito público era, hasta no hace mucho, aceptado como realidad innegable, asumido como natural debido a la fuerza social que posee el imaginario común basado en tópicos. 
Tal es el poder del estereotipo que, a través del proceso de socialización, "se encarnan en unos hábitos claramente diferentes de acuerdo con el principio de división dominante y capaces de percibir el mundo según ese principio" (Bourdieu, 2000: 38). Con el objetivo de romper esa dicotomía social, la escuela jugaría un papel fundamental al cuestionar los patrones de género que alumnos y alumnas poseen sobre hombres y mujeres, así como los roles que ambos juegan en la sociedad. El papel de la escuela sería el de ayudar a los alumnos a modificar esta percepción desde dos campos de intervención específicos: por una parte, el trabajo de deconstrucción de las ideas previas que posee el alumnado sobre las diferencias sexuales y, por otra, la adquisición de habilidades de relación más equitativas entre los géneros.

\section{La escuela ante el binomio sexo/género: posmodernismo, diferencia y Teoría Queer}

A principios de los años ochenta, al albor de la publicación de La Condition postmoderne: Rapport sur le savoir de Jean-François Lyotard (1979), se desarrolló un nuevo planteamiento teórico en el análisis de la desigualdad de las mujeres: el posmodernismo feminista, el cual realiza una crítica a los fundamentos modernos de base ilustrada. Dicha crítica se sustenta sobre el principio de poner en duda los conceptos de igualdad y razón y, para ello, se plantean las siguientes preguntas: ¿es posible conseguir la igualdad si somos diferentes?, ¿ existe alguna razón universal?

Finalmente, lo que se juzga es la existencia de algún tipo de criterio universal que integre a mujeres y varones. El posmodernismo afirma que los procesos universalizadores no atienden a la diversidad de contextos y situaciones de vida; por ello, la razón universalista siempre acaba tomando como referencia al hombre blanco, de clase económica media-alta, católico y de cultura occidental. Ante este principio rector del posmodernismo, cabe decir que autoras tan representativas del universalismo como Seyla Benhabib (2006) afirman que no se puede despreciar el pensamiento posmoderno, pues pone a prueba el legado del proyecto de la ilustración ayudando a mejorar sus postulados para resolver los problemas a los que las teorías de corte universal no han dado solución. En este sentido, no existe un único proyecto posmoderno sino distintas proyecciones y perspectivas. Esta autora habla de un posmodernismo fuerte y otro débil, el primero se reafirma en la idea de que el patriarcado ha muerto, y la versión débil destaca la importancia de la contextualización de los problemas y el punto de vista de las mujeres (Harding, 2010b); de 
ahí que se afirme que la versión débil podría ser una aliada del movimiento de mujeres (Benhabib, 2006: 242).

Sobre los cimientos del pensamiento posmoderno se alza la teoría de la "diferencia sexual", la cual ha realizado un desplazamiento respecto a las teorías posmodernas (Piussi y Mañeru, 2006: 15). Los postulados de la diferencia se han desarrollado ampliamente en Francia, donde destacan las obras de Luce Irigaray y Hélène Cixous (López, 2010); en Italia, Luisa Muraro y Anna María Piussi (Posada, 2005) son los pilares sobre los cuales se funda el pensamiento de la diferencia sexual.

Luce Irigaray es la representante más laureada del pensamiento de la diferencia; para ella el sexo es la categoría central de su análisis (Álvarez, 2001; Posada, 2006). Asimismo, para las demás autoras que sustentan esta posición teórica, la diferencia preponderante de todas las diferencias es la sexual; podríamos decirlo con su propio simbolismo: es la madre de todas las diferencias. En este sentido, se alejan del posmodernismo y del posestructuralismo. El hecho de poseer un cuerpo sexuado, construiría dos entes también distintos, que habría que potenciar en su especificidad. Para la Teoría de la diferencia, el genérico masculino se centra en la búsqueda del poder; en cambio, la femenina es la búsqueda del conocimiento y la relación. Esto se traduce en el ámbito educativo donde existiría una variable común sexual en la relación con el conocimiento. Tal y como señala la pedagoga más conocida de este movimiento: en las escuelas y en las universidades, donde cada vez hay mayor número de mujeres, éstas se moverían más por el deseo de conocer y compartir, que por la necesidad de conseguir un título académico (Piussi, 2001: 57; Arnaus y Piussi, 2010).

El objetivo educativo de esta perspectiva es resaltar y valorar positivamente las características que consideran ontológicamente femeninas. El primer paso es la exaltación de todo lo que tenga que ver con lo "femenino". Uno de los objetivos señalados por esta tendencia es que, desde el sistema educativo, se debe enseñar a la infancia la diferencia existente entre los sexos.

La práctica educativa que intenta fomentar se basa en la "relación" como principio de cualquier proceso educativo. Considera que la práctica de la relación es fundamentalmente femenina, pues, debido a la posibilidad de las mujeres de dar a luz, éstas poseen un cuerpo que "está abierto y disponible, en sí mismo, para acoger esa relación” (Arnaus, 2001: 231).

El conocimiento se entiende como un saber contextual, como experiencia, no como algo abstracto. Para ello, la práctica educativa se basará en la autorreflexibilidad, en la centralidad de la historia personal, en la experiencia 
global de vida, utilizando metodologías de aprendizaje narrativas, valorando positivamente las aportaciones de las chicas. Las especificidades en la práctica de la diferencia sexual se basan en potenciar los grupos diferenciados por sexos en el aula.

Las teorías Queer, a partir del posestructuralismo de Foucault y Derrida, están planteando también propuestas de reinterpretación de las teorías feministas, basándose en la búsqueda de la eliminación de la heteronormatividad, que, aseguran autoras como Judith Butler $(2001,2002)$, son la norma social sobre la cual se rige la identidad colectiva de nuestras sociedades. Es decir, se interpreta así: en la organización social la heterosexualidad funciona como norma que impide el desarrollo individual. Como oposición a esta situación, se propone la producción de diferencias eliminando la dualidad homosexualidad/heterosexualidad, abriendo el espacio social y de pensamiento analítico a nuevas formas de expresión de la identidad transexual (Butler, 2006: 89).

Para esta autora no existe diferencia entre sexo y género, pues considera que el sexo transciende lo biológico a una construcción cultural; lo ejemplifica en que, ya desde el mismo nacimiento, al observar el sexo de la persona recién nacida, se desarrolla una identidad previa a cualquier otra (Butler, 2001: 70). Desde esta perspectiva se está desarrollando un importante número de investigaciones en las escuelas sobre la sexualidad y cómo ésta crea y re-crea la identidad de la infancia y la juventud.

La diversidad de perspectivas en torno al género en el ámbito de la educación no significa que no existan principios básicos sobre los cuales se asienta una pedagogía, cuyo objetivo sea la búsqueda del desarrollo individual desde la superación de estereotipos basados en la oposición femenino-masculino y que determinan la vida de los seres humanos. Este objetivo aglutinador de las diferentes perspectivas se puede resumir en tres principios, que ya desde los años noventa se vienen proponiendo (Jackson, 1997: 457) y aún siguen vigentes:

1. Luchar por establecer relaciones igualitarias en el aula.

2. Intentar que todas y cada una de las personas de un aula sean valoradas como individuos.

3. Usar la experiencia del alumnado como un recurso didáctico en el proceso de enseñanza-aprendizaje.

\section{Consideraciones finales}

Las perspectivas teóricas señaladas han implementado un destacado impacto de género en el ámbito de la educación. Tal es así que la pedagogía ha cons- 
truido un fuerte campo de crítica androcéntrica, donde se ha cuestionado la neutralidad de los conocimientos tradicionales; es decir, la concepción según la cual el conocimiento está libre de valores y, en concreto, se ha analizado cómo las actuaciones pedagógicas tienden a orientarse en correspondencia con las percepciones y convicciones previas de las creencias asignadas a mujeres y varones. Por ello ha sido de vital importancia revisar los presupuestos científicos que revierten en la práctica educativa.

El hecho de que en el campo de la educación exista un amplio abanico de perspectivas teóricas en torno al género, ha posibilitado una potenciación de la actuación pedagógica en relación con su variedad de experiencias. Esta amalgama ha supuesto que en la práctica educativa existan posibilidades diversas de ejercer la docencia en función del contexto de las necesidades requeridas. Como hemos podido observar, cada una de las perspectivas planteadas pone el foco de atención en aquellos problemas que se consideran más relevantes para mejorar la situación de las mujeres en la sociedad a través de la educación.

La desigualdad es muy diversa y, según los contextos, afecta más a unos temas que a otros; por ejemplo, la importancia de que las niñas se incorporen a los centros escolares y obtengan una formación igual a la de sus compañeros varones es, en muchos lugares del planeta, el primero y más urgente de sus planteamientos, tal y como demanda la perspectiva liberal. Sabemos que es una cuestión cuantitativa y que la consecución de este objetivo no es suficiente para conseguir sociedades más equitativas, pero, sin ser suficiente, es más que necesario, es imprescindible.

La importancia de desterrar la docencia de su aliada: la feminidad tradicional, es un aspecto central para lograr que, tal y como demanda la perspectiva socialista, las mujeres no se vean abocadas a sobrellevar la doble y triple jornada. Hemos visto que los esfuerzos por poner en valor la profesión docente (que desarrollan mayoritariamente las mujeres) están vinculados con la imprescindible tarea de desligar este trabajo de la función maternal, pues se está induciendo que la docencia se enfrenta a los presupuestos de que ésta es una labor ejercida bajo imperativos biológicos y no científicos. La vinculación tradicional de la profesión docente como una actividad complementaria a la maternidad y el cuidado del hogar ha situado a las profesoras como el estandarte del aprovechamiento de la fuerza laboral de las mujeres a través de la segregación laboral, contribuyendo a la merma de su calidad de vida.

La perspectiva radical desarrolla un destacado trabajo en todas aquellas situaciones en que el cuerpo de la mujer posee una fuerte carga de cosificación 
y que se traduce en graves problemas de anorexia, falta de autoconfianza o interpretación de la violencia como un acto de amor sin cuestionar sus raíces de opresión. La situación de desigualdad viene dada por una opresión coercitiva y estructural basada en el patriarcado que, para esta perspectiva, la escuela debería problematizar, mediante procesos de autoconcientización de su situación de desigualdad como un primer paso, que dé lugar a que las propias mujeres reivindiquen su lugar en todos los órdenes de la estructura social.

Las aportaciones sobre la importancia de la valoración del cuerpo y la sexualidad, desde la interpretación que de ello realizan las propias mujeres, está siendo un campo de interpelación a la heteronormatividad que hace posible examinar en los centros educativos temáticas relacionadas con la identidad impuesta $v$ s identidad deseada. Este campo de trabajo que se ha desarrollado extraordinariamente en la última década con los estudios Queer venía precedido de un punto de partida que ha modificado grandes presupuestos teóricos, como ha sido el posestructuralismo en lo referente a su afán por desvelar las jerarquías sociales de oposiciones binarias, ejemplificadas en la oposición masculino/femenino.

Es imprescindible seguir debatiendo y abrir nuevos frentes de análisis de las prácticas y las teorías en relación con el género. Pero considero que debe hacerse desde planteamientos de comprensión y alianza, y no desde el enfrentamiento visceral con el cual, en muchas ocasiones, se debaten las diferentes propuestas planteadas en torno al género y su contribución a la educación. Conocer cada una de estas aportaciones ayudará a desarrollar un mayor abanico de propuestas pedagógicas, así como las mejores estrategias posibles en función del objetivo educativo que la realidad escolar le obligue a plantearse a cualquier docente que tenga como finalidad educativa la búsqueda de una sociedad más justa, basada en la libertad individual de "ser quien se quiera ser”, independientemente de la adscripción social de género.

\section{Bibliografía}

Álvarez, Silvina (2001), "Diferencia y teoría feminista”, en Beltrán, Elena y Virginia Maquieira [eds.], Feminismos. Debates teóricos contemporáneos, Madrid: Alianza.

Anderson, Elizabeth (2012), "Feminist Epistemology and Philosophy of Science", en Edward N. Zalta [ed.], The Stanford Encyclopedia of Philosophy, Stanford: Stanford University.

Arnaus, Remei (2001), “Aprender a lo largo de la vida desde el amor a la madre”, en Piussi, Ana y Ana Mañeru [coord.], Educación, nombre común femenino, Barcelona: Octaedro.

Arnaus, Remei y Anna Piussi [coord.] (2010), La Universidad fértil, Barcelona: Octaedro. 
Ana Sánchez-Bello. Perspectivas teóricas de género: status questionis del impacto en el sistema educativo

Benhabib, Seyla (2006), El ser y el otro en la ética contemporánea, Barcelona: Paidós.

Bourdieu, Pierre (2000), La dominación masculina, Barcelona: Anagrama.

Butler, Judith (2001), El género en disputa, Barcelona: Paidós.

Butler, Judith (2002), Cuerpos que importan. Sobre los limites materiales y discursivos del Sexo, Barcelona: Paidós.

Butler, Judith (2006), Deshacer el género, Barcelona: Paidós.

Coats, Maggie (1994), Women's Education, London: Open University Press.

Cobo, Rosa (2001), “Socialización e identidad de género. Entre el consenso y la coacción”, en Jornadas de Comunicación y Género, Málaga: CEDMA.

Connell, Robert William (2008), "Cool guys, swots and wimps: the interplay of masculinity and education”, en Sara Delamont and Paul Atkinson [eds.], Gender and Research, London: Sage.

Connell, Robert William (2001), Male roles, Masculinities and violence: a culture of peace perspective, Berkeley: United Nations Educational Scientific and Culture.

Eisenstein, Zillah (1980), Patriarcado capitalista y feminismo socialista, Madrid: Siglo XXI.

Flax, Jane (1981), Building feminist theory, New York: Lonngman.

Giner, Salvador et al. (1998), Diccionario de sociología, Madrid: Alianza.

Habermas, Jünger (1982), Conocimiento e interés, Madrid: Taurus.

Harding, Sandra (2002), “Strong Objectivity: A Response to the New Objectivity Question”, en Kourany, Janet [ed.], The Gender of Science, New Jersey: Prentice Hall.

Harding, Sandra (2010b), “Gendered standpoints on nature”, en Lorber, Judith, Gender inequality, New York: Oxford University Press.

Hobson, Barbara (2003), Recognition struggles and socialmovements, Cambridge: Cambridge University Press.

Holmstrom, Nancy (2002), The Socialist Feminist Project: A Contemporary Reader in Theory and Politics, New York: Monthly Review Press.

Jackson, Sue (1997), “Crossing borders and changing pedagogies: from Giroux and Freire to feminist theories of education", en Gender and Education, vol. 9, núm. 4, UK: Routledge.

Jagar, Alison (1983), Feminist, Politics and Human Nature, Totowa: N. J. Rowman and Allanheld.

Kolmar, Wendy y Frances Bartkowski (2010), Feminist theory, Boston: McGraw Hill.

Longino, Helen (2002), The Fate Of Knowledge, Princeton: Princeton University Press.

López, Mercedes (2010), “Una simbología otra del cuerpo de la mujer: la apertura al otro”, en Investigaciones Fenomenológicas, vol. monográfico 2, Madrid: UNED.

Lorber, Judith (2010), Gender inequality, New York: Oxford University Press.

Lyotard, Jean-François (1979), La condition postmoderne: rapport sur le savoir, Paris: Minuit.

Mañeru, Ana (2001), "La diferencia sexual en la educación”, en Blanco, Nieves, Educar en femenino y en masculino, Madrid: Akal. 
Piussi, Anna (2001), "El sentido libre de la diferencia sexual", en Cuadernos de Pedagogía, núm. 306, Barcelona: Praxis.

Piussi, Ana y Ana Mañeru (2006), Educación, nombre común femenino, Barcelona: Octaedro.

Posada, Luisa (2005), "El pensamiento de la diferencia sexual: el feminismo italiano", en Amorós, Celia y Ana De Miguel, Teoría feminista: de la ilustración a la globalización, Madrid: Minerva.

Posada, Luisa (2006), "Diferencia, identidad y feminismo: una aproximación al pensamiento de Luce Irigaray", en Logos. Anales del Seminario de Metafísica, vol. 39, Madrid: Universidad Complutense.

Renau, Dolors (2012), Cómo aprender a amar en la escuela, Madrid: Catarata.

Sánchez, Cristina et al. (2001), "Feminismo liberal, radical y socialista", en Beltrán, Elena y Virginia Maquieira [eds.], Feminismos. Debates teóricos contemporáneos, Madrid: Alianza.

Skelton, Christine y Becky Francis (2009), Feminism and the schooling sacandal, London: Routledge.

Totten, Samuel y Jon Pedersen (2012), Educating about social issues in the 20th and 21st centuries, USA: Library of Congress.

Weiner, Gaby (1999), Los feminismos en la educación, Morón: Kikiriki.

Wood, Julia (2011), Gendered lives. Communication, gender and culture, Boston: Wadswoth.

\section{Recursos electrónicos}

Harding, Sandra (2010a), "Gender, democracy and philosophy of science", en The pantaneto Forum, núm. 38, London: Pantaneto Forum. Disponible en: http://www.pantaneto. co.uk/issue38/harding.htm [25 de noviembre de 2012].

Ministerio de Educación de España (2010), Informe sobre el estado situación, Madrid: CIDE. Disponible en: www.educacion.gob.es/horizontes/estadisticas.html [3 de noviembre de 2012].

Ana Sánchez Bello. Doctora en Psicopedagogía y profesora de Diseño, desarrollo y evaluación curricular en la Universidad de La Coruña. Ha colaborado como docente en las universidades de Oporto, Granada y Castellón. Es miembro del Grupo de Investigación en Innovaciones Educativas (GIE) y participa de diversos proyectos de investigación de concurrencia competitiva, vinculados con el Ministerio de Educación, desde donde desarrolla sus líneas de investigación centradas en las mujeres en la ciencia y políticas de igualdad. Publicaciones recientes: "Gendered agenda in education", en Globalism and power: Iberian Education and Curriculum Policies, New York: Peter Lang (2012); "Gender violence and leisure in Spain: looking for new ways in educational research", en Educaço, sociedade \& culturas, Oporto: Universidad de 
Ana Sánchez-Bello. Perspectivas teóricas de género: status questionis del impacto en el sistema educativo

Oporto (2012); "Sex education representations in spanish combined biology and geology textebooks", en International Journal of Science Education, disponible en: <www.tandfonline.com/loi/tsed20> (2013).

Recepción: 5 de junio de 2013.

Aprobación: 23 de junio de 2014. 
